\title{
Factors Related to the Development of Shunt-Dependent Hydrocephalus Following Subarachnoid Hemorrhage in the Elderly
}

\author{
Tae Seok JEONG, Chan Jong YOO, Woo Kyung KIM, Gi Taek YEE, Eun Young KIM, Myeong Jin KIM \\ Ga-chon University, Gil Medical Center, Department of Neurosurgery, Incheon, Korea
}

\section{ABSTRACT}

AIM: Surgical procedures for aneurysmal subarachnoid hemorrhage (SAH) are increasing among the elderly as the population ages. Chronic shunt-dependent hydrocephalus is a recognized complication of SAH. The aim of this study was to identify predictive factors for the development of shunt-dependent hydrocephalus among elderly patients with SAH.

MATERIAL and METHODS: We retrospectively studied 878 patients, including 275 patients $\geq 65$ years old, with SAH treated between 2005 and 2015 to identify factors contributing to the development of shunt-dependent hydrocephalus. The relationships between shunt-dependent hydrocephalus and the causative factors were analyzed using univariate and multivariate analysis; the causative factors were based on the results of previous studies.

RESULTS: In the 878 patients with SAH, there was a significant difference in the incidence of shunt-dependent hydrocephalus between patients $<65$ years old and those $\geq 65$ years old $(p=0.021)$. In the 275 patients $\geq 65$ years old, the following were associated with shunt-dependent hydrocephalus on univariate analysis: 1) Hunt and Hess grade ( $p=0.005)$, 2) Fisher grade $(p<0.001), 3)$ intraventricular hemorrhage $(p<0.001), 4)$ acute hydrocephalus $(p=0.003)$, 5) aneurysm location $(p=0.001)$, and 6$)$ external ventricular drain placement $(p<0.001)$. On multivariate analysis, only 1$)$ intraventricular hemorrhage $(p<0.001)$ and 2$)$ a ruptured aneurysm located in the distal posterior circulation $(p=0.014)$ were related to an increased risk for the development of shunt-dependent hydrocephalus.

CONCLUSION: Evaluating risk factors can help identify patients at high risk of developing shunt-dependent hydrocephalus. Identifying these risk factors may help neurosurgeons to provide optimal therapy and improve outcomes in patients with SAH.

KEYWORDS: Aneurysm, Shunt, Geriatrics, Hydrocephalus, Subarachnoid hemorrhage

\section{INTRODUCTION}

$\mathrm{H}$ ydrocephalus, a well-known complication of aneurysmal subarachnoid hemorrhage $(\mathrm{SAH})$, generally has been divided into three stages: acute (0-3 days after $\mathrm{SAH})$, subacute (4-13 days after $\mathrm{SAH})$, and chronic $(\geq$ 14 days after $\mathrm{SAH}$ ) (25). Hydrocephalus occurring during the acute or subacute stage is commonly treated with an external ventricular drain (EVD) and a shunt procedure is performed if hydrocephalus develops when the drainage bag is clamped or elevated after a certain period. In cases that develop hydrocephalus weeks or months after $\mathrm{SAH}$, shunt surgery is indicated. The incidence of shunt-dependent hydrocephalus in $\mathrm{SAH}$ patients has been reported to range from 6 to $37 \%(21)$.

Several studies have reported on those factors related to the development of shunt-dependent hydrocephalus following $\mathrm{SAH}(8,10,21-24,26)$. Although there are some differences in the reported risk factors, several factors have been commonly recognized as significant including advanced age, acute 
hydrocephalus, aneurysm location in the posterior circulation, intraventricular hemorrhage (IVH), severe bleeding visualized with brain computed tomography (CT), and poor clinical status at admission (15).

The frequency of SAH is three- to four-fold higher in elderly patients (2). In South Korea, there has been a noticeable increase in SAH in elderly patients because of the rapidly aging population. Elderly patients usually have more underlying diseases and may have had longer exposures to tobacco and alcohol, which can affect the course of SAH to a greater extent than that seen in young patients.

The purpose of this study was to identify predictive factors for the development shunt-dependent hydrocephalus among elderly patients with SAH.

\section{MATERIAL and METHODS}

\section{Patient Population}

We retrospectively studied 878 consecutive patients who underwent surgical treatment for ruptured aneurysmal SAH in our institution between 2005 and 2015. We previously excluded 283 patients (death=259, lost to follow-up=24) from the total number of 1161 patients with $\mathrm{SAH}$ treated during that time period. Of the 878 patients with SAH, 603 patients were < 65 years old and 275 patients were $\geq 65$ years old.

All patients with SAH underwent surgical procedure within 12 hours of admission and treatment was based on the size, morphology, location, and the amount of bleeding associated with the aneurysm as shown by brain CT, CT-angiography, and digital subtraction angiography.

Acute hydrocephalus was diagnosed if the modified Evan's ratio was > 0.31 on the initial brain CT scan (11). An EVD procedure was performed when the neurological status at admission was poor, or when imaging studies revealed acute hydrocephalus or findings of increased intracranial pressure (IICP) or brain edema, such as ventricular dilatation, sulcus obliteration, slit ventricle, and periventricular lucency. Brain CT was performed immediately after surgery (clipping or coiling) and a follow-up brain CT was performed before discharge. For patients who underwent EVD or had a poor clinical course, serial CT follow-up was performed. If hydrocephalus progressed postoperatively in patients without EVD, an EVD procedure was performed. The EVD catheter was removed if hydrocephalus did not progress after the drip chamber was elevated or clamped. A ventriculoperitoneal shunt was performed if hydrocephalus persisted. Outpatient followup was performed at 2 weeks, 1, 3, 6, and 12 months, and annually post discharge. If there was radiological evidence of enlarged ventricles, periventricular lucencies, rounding of the frontal horns, or obliteration of the cerebral sulci on follow-up brain CT along with symptoms, such as chronic headache, walking difficulty, poor memory, and bladder problems judged to be caused by hydrocephalus, a shunt procedure was performed after assessing for symptom improvement with a lumbar puncture.

\section{Analysis of Data}

Initially, all the patients were divided into two groups: age < 65 years old and age $\geq 65$ years old. Because there was a higher frequency of the shunt procedure in the later group, we analyzed the patients' characteristics of that group to identify risk factors. Patients $\geq 65$ years old were divided into two groups according to whether they required a shunt procedure. Predictive factors were analyzed based on those factors shown to influence shunt-dependent hydrocephalus in previous studies $(8,21,23)$ including age, sex, Hunt and Hess grade, Fisher grade, IVH, acute hydrocephalus, aneurysm location, treatment type, EVD placement, postoperative central nervous system (CNS) infection, and postoperative hematoma. In addition, factors, such as underlying disease, smoking history, and alcohol intake were evaluated.

\section{Statistical Analysis}

The relationship between predictive factors and the development of shunt-dependent hydrocephalus was analyzed in two steps. Univariate analysis for all predictive factors was performed using the chi-square test, and for those parameters shown to have a $p$ value $<0.1$ on univariate analysis, multivariate analysis was carried out using logistic regression. Age was analyzed with the independent t-test. All tests were performed by using $a=0.05$, and the SPSS for Windows Version 23.0 statistical package (SPSS, Inc., Chicago, IL) was used for all analyses.

\section{- RESULTS}

\section{Comparison of patients under and over age 65}

Out of 878 patients with SAH overall, shunt surgery was performed in $13.3 \%$ of patients < 65 years old and in $19.3 \%$ of patients $\geq 65$ years old. This difference was statistically significant $(\mathrm{p}=0.021)$ and the odds ratio $(\mathrm{OR})$ for shunt surgery in those patients $\geq 65$ years old was 1.56 (Table I).

\section{Patients aged 65 years and older}

\section{Non-shunt-treated group versus shunt-treated group}

A total of 275 patients $\geq 65$ years old were divided into two groups (non-shunt-treated group, 222; shunt-treated group, 53). The mean patient age was 70.6 years and there was no significant difference in age between the two groups (Table II).

\section{Sex}

Patients $\geq 65$ years old consisted of 220 females $(80 \%)$ and 55 males (20\%). The sex ratio between those patients requiring shunts and those that did not require shunts was not significantly different (Table II). Multivariate analysis was carried out because the $p$ value for sex was $<0.1$ ( $p=0.093$, and there was no significant difference in shunt requirement between females and males ( $p=0.264$ ) (Table III).

\section{Neurological Status at Admission}

Neurological status at admission was evaluated using the Hunt and Hess grade. On univariate analysis with the chi-square test, there was a significant difference between those patients 
Table I: Comparison of Shunt Procedure Between Patients $<65$ Years Old and Patients $\geq 65$ Years Old

\begin{tabular}{ccccc}
\hline & $\begin{array}{c}\text { Group }<\text { 65 years old } \\
(\mathbf{n = 6 0 3 )}\end{array}$ & $\begin{array}{c}\text { Group } \geq 65 \text { years old } \\
(\mathbf{n = 2 7 5 )}\end{array}$ & $\begin{array}{c}\text { Total } \\
(\mathbf{n = 8 7 8})\end{array}$ & OR value \\
\hline Shunt procedure & & & & 1.56 \\
\hline - No & $523(86.3 \%)$ & $222(80.7 \%)$ & $745(84.9 \%)$ & $0.021^{*}$ \\
\hline - Yes & $80(13.3 \%)$ & $53(19.3 \%)$ & $133(15.1 \%)$ \\
\hline
\end{tabular}

"Statistically significant differences ( $p$ value $<0.05)$, OR: odds ratio.

Table II: Factors Related to Shunt-Dependent Hydrocephalus Following SAH

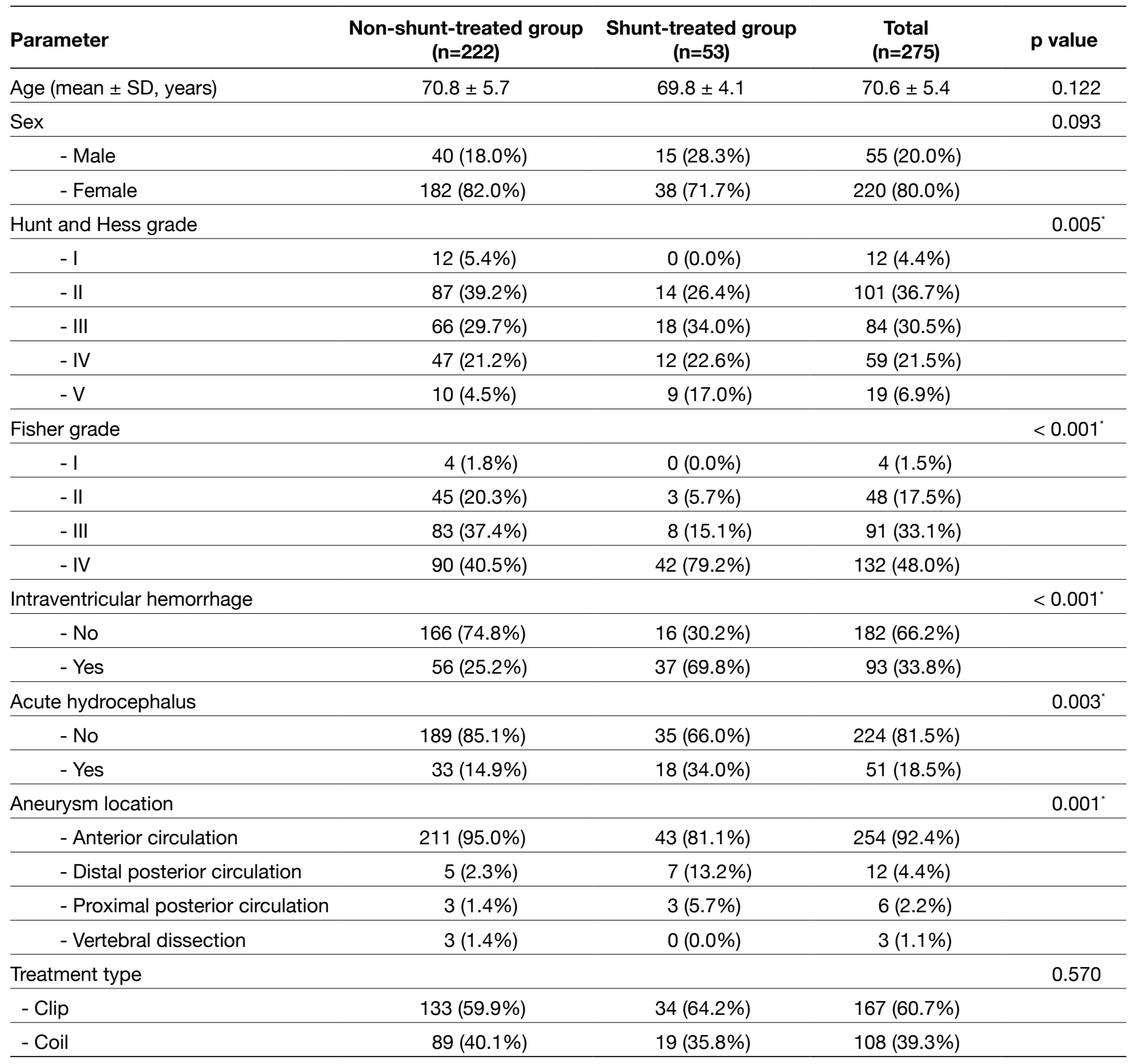


Table II: Cont.

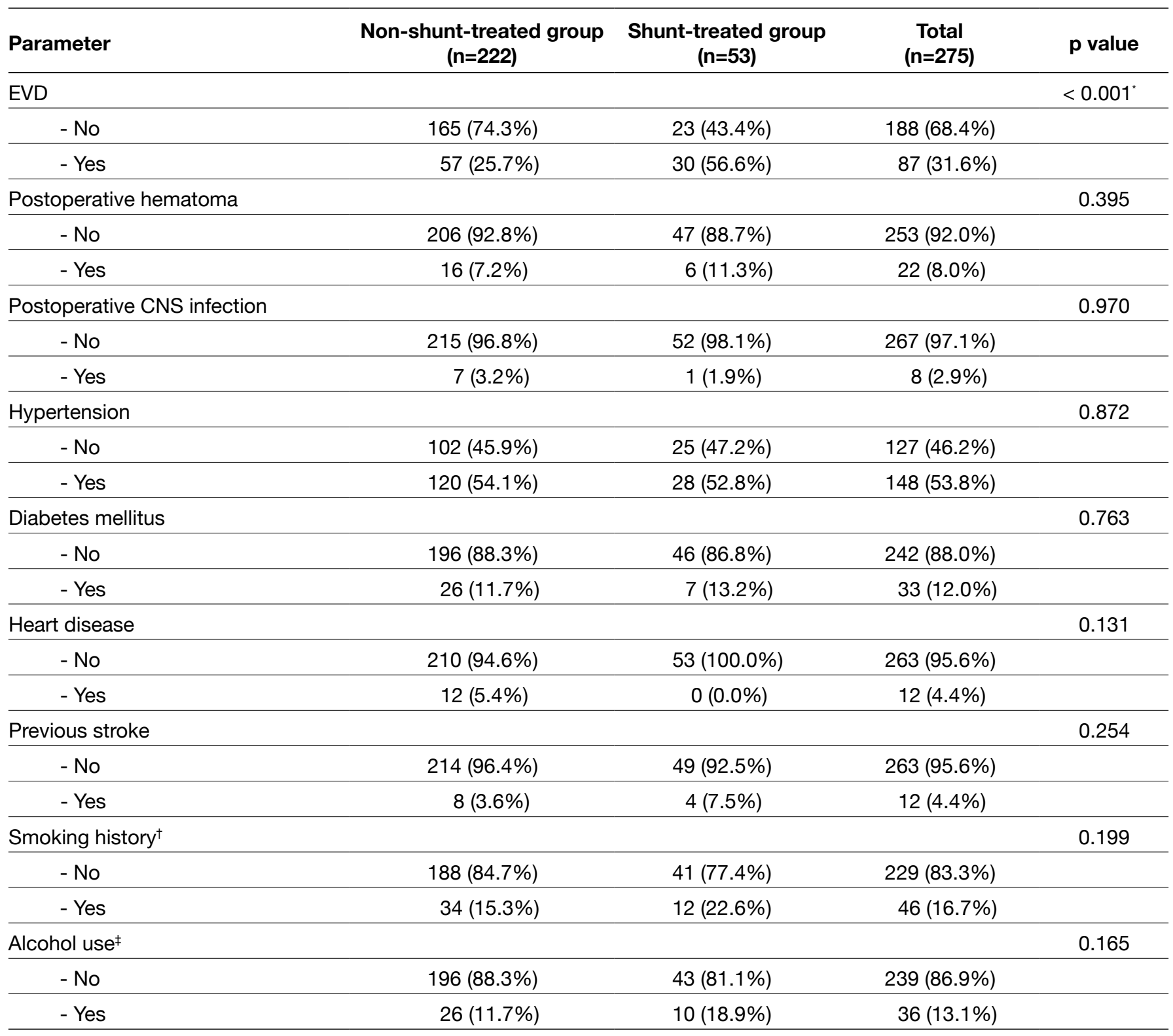

* Statistically significant differences $(p$ value $<0.05)$

+ Current or former smoker

*Alcohol consumption more than twice per week

CNS: Central nervous system, EVD: External ventricular drainage, SAH: Subarachnoid hemorrhage, SD: Standard deviation.

$\geq 65$ years old requiring shunts and those that did not require shunts $(p=0.005)$ (Figure 1, Table II). On multivariate analysis with logistic regression, Hunt and Hess grades were divided into low (I, II, and III) and high (IV and V) grades according to the survival rate of each group reported by Hunt and Hess (16). Neither patient group showed a significant difference for the low and high Hunt and Hess grades $(p=0.348)$ (Table III).

\section{Amount of Subarachnoid Hemorrhage}

The amount of SAH was evaluated by using the Fisher grade on the initial brain CT scan at admission. On univariate analysis, most patients $\geq 65$ years old requiring shunts and those $\geq 65$ years old that did not require shunts were Fisher grade 4 , but the overall Fisher grade was significantly higher in the shunt-treated group $(p<0.001)$ (Figure 2, Table II). However, on multivariate analysis, Fisher grades were divided into low (grades 1 and 2) and high (grades 3 and 4) grades, and neither patient group showed a significant difference $(p=0.371)$ (Table III).

\section{Presence of Intraventricular Hemorrhage}

The presence of IVH and the development of shunt-dependent 
Table III: Multivariate Analysis of Potential Risk Factors for ShuntDependent Hydrocephalus (Logistic Regression Method)

\begin{tabular}{lcc}
\hline & $\begin{array}{c}\text { Adjusted } \\
\text { OR }\end{array}$ & p value \\
\hline Sex & & \\
\hline \multicolumn{1}{l}{ - Male } & 1.00 & \\
\hline - Female & 0.63 & 0.264 \\
\hline Hunt and Hess grade & & \\
\hline - Low (I, II, III) & 1.00 & \\
\hline - High (IV, V) & 0.66 & 0.348 \\
\hline Fisher grade & & \\
\hline - Low (I, II) & 1.00 & \\
\hline - High (III, IV) & 1.86 & 0.371 \\
\hline Intraventricular hemorrhage & 5.28 & $<0.001^{*}$ \\
\hline Acute hydrocephalus & 1.15 & 0.749 \\
\hline Aneurysm location & & \\
\hline & & \\
\hline - Anterior circulation & 1.00 & \\
\hline - Distal posterior circulation & 5.84 & $0.014^{*}$ \\
\hline - Proximal posterior circulation & 3.17 & 0.233 \\
\hline & & \\
\hline & & \\
\hline & & \\
\hline
\end{tabular}

"Statistically significant differences ( $p$ value $<0.05)$

EVD: external ventricular drainage, OR: odds ratio.

hydrocephalus showed a significant relationship on univariate analysis $(\mathrm{p}<0.001)$ (Table II). Also, on multivariate analysis, the shunt-treated group had a significantly higher risk of IVH compared to the non-shunt-treated group $(p<0.001$, adjusted $\mathrm{OR}=5.28$ ) (Table III).

\section{Acute Hydrocephalus at Admission}

Acute hydrocephalus was diagnosed radiologically using the Evan's ratio on the initial brain CT scan at admission. Imaging studies that did not meet acceptable criteria were excluded from the analysis. On univariate analysis, the percentage of patients with acute hydrocephalus was low (18.5\%), but it was significantly higher in the shunt-treated group (34\%) compared with that of the non-shunt-treated group (14.9\%) $(p=0.003)$ (Table II). However, on multivariate analysis, there was no significant difference between the two patient groups $(p=0.749)$ (Table III).

\section{Aneurysm Location}

Aneurysm location was evaluated using the four location categorizations documented by Dorai et al. (8): 1) anterior circulation (anterior communicating artery, internal carotid

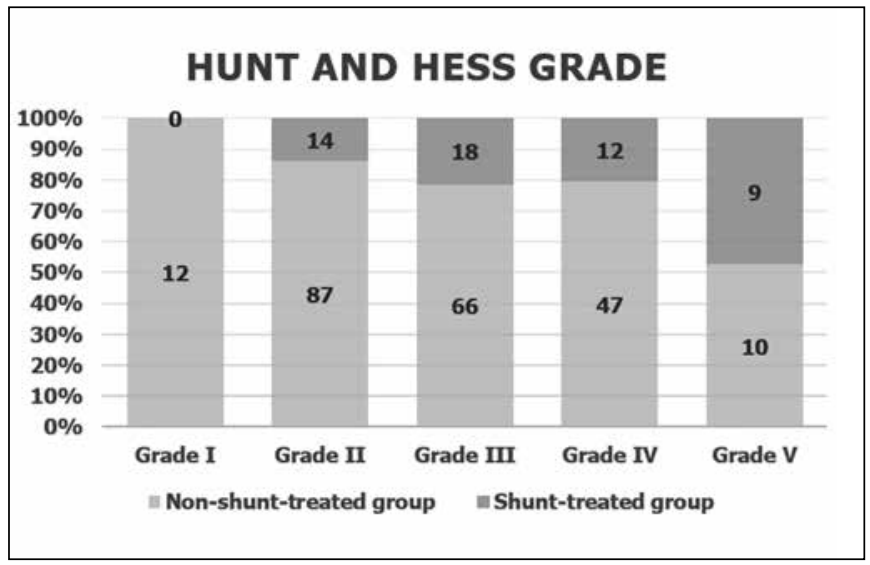

Figure 1: Hunt and Hess grade and percentages of non-shunttreated and shunt-treated patients in each grade.

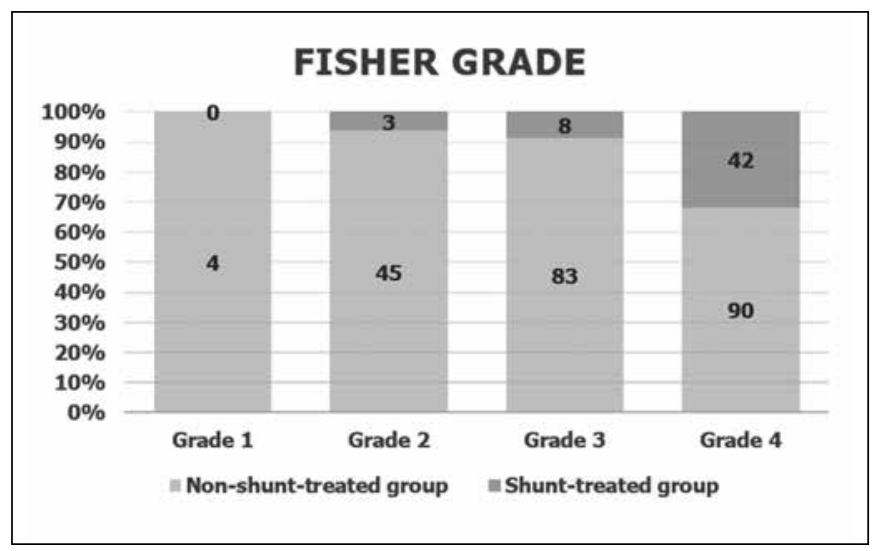

Figure 2: Fisher grade and percentages of non-shunt-treated and shunt-treated patients in each grade.

artery, and middle cerebral artery), 2) proximal posterior circulation (posteroinferior cerebellar artery, vertebral confluence, and anteroinferior cerebellar artery), 3) distal posterior circulation (basilar artery, posterior cerebral artery, and superior cerebellar artery), and 4) vertebral dissections.

Anterior circulation lesions accounted for most of the aneurysm locations in patients $\geq 65$ years old requiring shunts and in those patients $\geq 65$ years old that did not require shunts. However, there were significant differences between the groups on univariate analysis $(p=0.001)$ (Table II). On multivariate analysis, the distal posterior circulation differed significantly in the shunt-treated group $(p=0.014)$ with an OR of 5.84 compared with the anterior circulation as a reference (Table III).

\section{Clip versus Coil Treatment}

Treatment type (clipping or coiling) was determined based on the location and shape of the aneurysm, brain edema, and surgeon preference. Overall, clipping $(60.7 \%)$ was performed more frequently than coiling $(39.3 \%)$, but there were no significant differences between the two patient groups (Table II). 


\section{External Ventricular Drain Placement}

Patients who underwent an EVD procedure during hospitalization period were evaluated, though data regarding the duration and frequency of EVD were not available. On univariate analysis, there was a significant difference between patients $\geq$ 65 years old requiring shunts and those $\geq 65$ years old that did not require shunts $(p<0.001)$ (Table II). On multivariate analysis, EVD placement showed a trend towards an increasing incidence in shunt-treated patients, but this difference was not statistically significant $(p=0.102)$ (Table III).

\section{Postoperative Complications}

Postoperative complications, including hematoma and CNS infection, were evaluated as possible risk factors for the development of chronic hydrocephalus. Postoperative hematoma was diagnosed when new intracerebral hemorrhage (ICH) or IVH was observed or when the amount of SAH distinctly increased on the immediate postoperative brain CT scan compared with the preoperative CT. There were no significant differences in the incidence of postoperative hematoma and CNS infection between patients $\geq 65$ years old requiring shunts and those $\geq 65$ years old that did not require shunts (Table II).

\section{Underlying diseases, smoking, and alcohol abuse}

Underlying diseases, including hypertension (HTN), diabetes mellitus (DM), heart disease, and previous stroke, which may be more prevalent in older patients, were evaluated. Also, smoking and alcohol abuse were investigated. There were no significant differences between the patient groups in any of these factors (Table II).

\section{DISCUSSION}

Hydrocephalus is a well-known complication of SAH. Several mechanisms have been proposed to explain the development of hydrocephalus among patients after $\mathrm{SAH}$, and several theories suggest a role for alterations in cerebrospinal fluid (CSF) dynamics $(1,4,7,8,14)$. Hydrocephalus may occur via obstructive mechanisms when blood products or adhesions block CSF circulation within the ventricular system $(12,25)$ or may result from absorptive problems attributable to impaired CSF absorption at the arachnoid granulations $(5,6,9)$.

Our study showed that patients $\geq 65$ years old had a higher incidence of shunt dependency than younger patients. Numerous theories have been postulated to explain the frequency of shunt dependence in the elderly. With wider subarachnoid spaces, older patients can hold larger amounts of subarachnoid blood, thus increasing their risk of developing CSF circulation disturbances (26). Also, with age, the extent of meningeal fibrosis increases, leading to impaired CSF circulation and decreased CSF absorption (19). Also, ventricular compliance decreases, which may explain why hydrocephalus among elderly patients is more likely to be symptomatic (20).

Besides increasing age, various other factors that might influence the frequency of shunt-dependent hydrocephalus have been suggested by previous studies $(8,10,21-24,26)$. In our study, we analyzed these factors in elderly patients and found that IVH and aneurysms located in the distal posterior circulation were significantly associated factors. The importance of these two factors was suggested by the findings of previous studies. However, sex, treatment type, postoperative hematoma, and CNS infection had no relationship with the development of shunt-dependent hydrocephalus. Additionally, Hunt and Hess grade, Fisher grade, acute hydrocephalus, and EVD placement were significant on univariate analysis, but were not found to be significant on multivariate analysis.

Our study showed that IVH was the predictive factor with the highest OR. IVH is known to be a risk factor for both acute and chronic hydrocephalus (25), and we often anticipate that patients with $\mathrm{SAH}$ and IVH will develop acute hydrocephalus and will have a high Fisher grade and a poor neurological status. Finally, it is thought that significant factors on univariate analysis became insignificant on the multivariate analysis because of the correlations between these factors.

Treatment type had no significant influence on the likelihood of the development of shunt-dependent hydrocephalus in this study. Dorai et al. (8) reported that the incidence of shuntdependent hydrocephalus was lower in cases of clipping than in coiling because of irrigation of SAH clots and fenestration of the lamina terminalis during clipping. However, Komotar et al.(17) reported that fenestration of the lamina terminalis might not reduce the incidence of shunt-dependent hydrocephalus. Also, the relationship between coiling and aneurysm location was not analyzed in the study by Dorai et al. (8). In the clipping procedure, the approach for clipping an anterior circulation aneurysm is relatively easy, but the approach for clipping a posterior circulation aneurysm is more difficult. Therefore, coiling is preferred over clipping in many cases of a posterior circulation aneurysm. There is a possibility that coiling was found to be a risk factor on univariate analysis because posterior circulation aneurysms are a risk factor for shunt-dependent hydrocephalus. As coiling techniques have improved recently, many middle cerebral artery aneurysms previously treated with clipping have now been treated with a coil. In our institution, treatment with clipping was more frequent than treatment with a coil in the past, but recently there is a trend to treat with coiling as opposed to clipping. Therefore, considering the relationship of treatment to aneurysm location, it is thought that treatment type has no significant influence on the development of shunt-dependent hydrocephalus.

Patients with acute hydrocephalus after SAH often need to undergo EVD during the acute stage to control increased ICP or to relieve brain edema. Although it is controversial if EVD placement is related to the development of shunt-dependent hydrocephalus, some reports have provided evidence that the duration of EVD is a statistically significant predictor for the development of chronic hydrocephalus after SAH $(1,13,16)$. Continuous CSF drainage via EVD could promote the occlusion of normal CSF pathways. Intentional intermittent CSF drainage is recommended to keep the normal subarachnoid pathways 
open because CSF pulse pressure is an important force that helps to prevent the formation of occlusive membranes and clots $(1,13)$. In our study, EVD placement was associated with a high incidence of shunt-dependent hydrocephalus on univariate analysis, though not on multivariate analysis. We believe that the duration of EVD usage was about 1 2 weeks, and that it may have affected CSF pathways.

Tapaninaho et al.(23) reported that postoperative hematoma and infection have a positive influence on the development of shunt-dependent hydrocephalus but that study included all types of infection, not just CNS infections. CNS infection is a well-known cause of hydrocephalus (3). However, our study found that postoperative CNS infection did not have a statistically significant effect on the development of shunt dependent hydrocephalus. This may be because of the small number of infections that occurred in our study.

We also investigated factors such as HTN, DM, heart disease, and previous stroke, which are more frequent in elderly patients, and may affect the cerebrovascular system. However, none of these factors was associated with a statistically significant difference. Previous studies have evaluated the impact of smoking and alcohol on the cerebrovascular system. Lasner et al. (18) reported that smoking increased the risk of vasospasm after SAH, and Sheehan et al. (22) reported that alcohol abuse increased the incidence of hydrocephalus after $\mathrm{SAH}$. In our study, both smoking and alcohol use were more frequently present in the shunt-dependent hydrocephalus group, but the differences were not significant. A larger study may have provided more significant findings.

There are several limitations of our study. There were few relatively cases in the $\geq 65$ year old shunt-dependent group. We believe that some factors might have been found to be significant if the number of cases was larger. Also, we did not evaluate for vasospasm as a risk factor as suggested by several previous reports $(8,26)$. We excluded vasospasm from the group of factors to be analyzed because data for vasospasm was incomplete in our retrospective study design. We also did not consider the duration of EVD in our analysis. To evaluate EVD more precisely as a risk factor for the development of chronic hydrocephalus after $\mathrm{SAH}$, additional analysis for the duration of EVD is needed.

Several reports have documented the risk factors for the development of shunt-dependent hydrocephalus, and some factors have been commonly accepted as risk factors. We investigated these factors to identify specific factors in elderly patients. Nonetheless, our results showed that these risk factors differ minimally between younger and older patients. Although new risk factors were not identified in our study, we were able to verify that IVH and aneurysms located in the distal posterior circulation had a strong relationship with the development of shunt-dependent hydrocephalus in elderly patients.

\section{CONCLUSION}

Shunt-dependent hydrocephalus in patients with SAH is a serious complication requiring additional surgery as well as treatment for the aneurysm. The development of shuntdependent hydrocephalus has a negative effect on patient outcome, especially in elderly patients. Assessing the risk factors for shunt-dependent hydrocephalus can help identify patients with a high risk of developing shunt-dependent hydrocephalus, and this may assist neurosurgeons in optimizing therapy and improving the outcomes of patients with SAH. To minimize bias and clearly identify risk factors of shuntdependent hydrocephalus, future randomized controlled trials are needed.

\section{- REFERENCES}

1. Auer LM, Mokry M: Disturbed cerebrospinal fluid circulation after subarachnoid hemorrhage and acute aneurysm surgery. Neurosurgery 26: 804-808; discussion 808-809, 1990

2. Awe OO, Gonzalez LF, Hasan D, Maltenfort M, Rossenwasser R, Jabbour P: Treatment outcome of aneurysmal subarachnoid hemorrhage in patients aged 70 years and older. Neurosurgery 68: 753-758, 2011

3. Beni-Adani L, Biani N, Ben-Sirah L, Constantini S: The occurrence of obstructive vs absorptive hydrocephalus in newborns and infants: Relevance to treatment choices. Childs Nerv Syst 22: 1543-1563, 2006

4. Black PM, Tzouras A, Foley L: Cerebrospinal fluid dynamics and hydrocephalus after experimental subarachnoid hemorrhage. Neurosurgery 17: 57-62, 1985

5. Blasberg R, Johnson D, Fenstermacher J: Absorption resistance of cerebrospinal fluid after subarachnoid hemorrhage in the monkey; Effects of heparin. Neurosurgery 9: 686-691, 1981

6. Brydon HL, Bayston R, Hayward R, Harkness W: The effect of protein and blood cells on the flow-pressure characteristics of shunts. Neurosurgery 38: 498-504; discussion 505, 1996

7. Doczi T, Nemessanyi Z, Szegvary Z, Huszka E: Disturbances of cerebrospinal fluid circulation during the acute stage of subarachnoid hemorrhage. Neurosurgery 12: 435-438, 1983

8. Dorai Z, Hynan LS, Kopitnik TA, Samson D: Factors related to hydrocephalus after aneurysmal subarachnoid hemorrhage. Neurosurgery 52: 763-771, 2003

9. Ellington E, Margolis G: Block of arachnoid villus by subarachnoid hemorrhage. J Neurosurg 30: 651-657, 1969

10. Fülöp B, Deak G, Mencser Z, Kuncz A, Barzó P: Factors affecting the development of chronic hydrocephalus following subarachnoid hemorrhage, with special emphasis on the role of ventricular and lumbar drainage. Ideggyogy Sz 62: 255261, 2009 (In Hungarian)

11. Gyldensted C: Measurements of the normal ventricular system and hemispheric sulci of 100 adults with computed tomography. Neuroradiology 14: 183-192, 1977

12. Hasan D, Tanghe HL: Distribution of cisternal blood in patients with acute hydrocephalus after subarachnoid hemorrhage. Ann Neurol 31: 374-378, 1992

13. Hirashima $Y$, Hamada H, Hayashi N, Kuwayama N, Origasa $\mathrm{H}$, Endo S: Independent predictors of late hydrocephalus in patients with aneurysmal subarachnoid hemorrhage-analysis by multivariate logistic regression model. Cerebrovasc Dis 16: 205-210, 2003 
14. Hiratsuka H, Suganuma Y, Tsuyumu M, Oata M: Radioisotope cisternographic study on cerebrospinal fluid circulation after subarachnoid hemorrhage. No Shinkei Geka 3: 139-144, 1975 (In Japan)

15. Hunt WE, Hess RM: Surgical risk as related to time of intervention in the repair of intracranial aneurysms. J Neurosurg 28: 14-20, 1968

16. Kasuya H, Shimizu T, Kagawa M: The effect of continuous drainage of cerebrospinal fluid in patients with subarachnoid hemorrhage: A retrospective analysis of 108 patients. Neurosurgery 28: 56-59, 1991

17. Komotar R, Hahn D, Kim G, Khandji J, Mocco J, Mayer S, Connolly $E$ Jr: The impact of microsurgical fenestration of the lamina terminalis on shunt-dependent hydrocephalus and vasospasm after aneurysmal subarachnoid hemorrhage. Neurosurgery 62: 123, 2008

18. Lasner TM, Weil RJ, Riina HA, King JT Jr, Zager EL, Raps EC, Flamm ES: Cigarette smoking-induced increase in the risk of symptomatic vasospasm after aneurysmal subarachnoid hemorrhage. J Neurosurg 87: 381-384, 1997

19. Mehta V, Holness RO, Connolly K, Walling S, Hall R: Acute hydrocephalus following aneurysmal subarachnoid hemorrhage. Can J Neurol Sci 23: 40-45, 1996
20. Niizuma H, Yoshimoto T, Suzuki J: Direct operation of cerebral aneurysms over 70 years of age. Jpn J Stroke 7: 219-223, 1985

21. O'Kelly CJ, Kulkarni AV, Austin PC, Urbach D, Wallace MC: Shunt-dependent hydrocephalus after aneurysmal subarachnoid hemorrhage: Incidence, predictors, and revision rates: Clinical article. J Neurosurg 111: 1029-1035, 2009

22. Sheehan JP, Polin RS, Sheehan JM, Baskaya MK, Kassell NF: Factors associated with hydrocephalus after aneurysmal subarachnoid hemorrhage. Neurosurgery 45: 1120-1127; discussion 1127-1128,1999

23. Tapaninaho A, Hernesniemi J, Vapalahti M, Niskanen M, Kari A, Luukkonen M, Puranen M: Shunt-dependent hydrocephalus after subarachnoid haemorrhage and aneurysm surgery: Timing of surgery is not a risk factor. Acta Neurochir 123: 118124, 1993

24. Vale FL, Bradley EL, Fisher III WS: The relationship of subarachnoid hemorrhage and the need for postoperative shunting. J Neurosurg 86: 462-466, 1997

25. van Gijn J, Hijdra A, Wijdicks EF, Vermeulen M, van Crevel $\mathrm{H}$ : Acute hydrocephalus after aneurysmal subarachnoid hemorrhage. J Neurosurg 63: 355-362, 1985

26. Yoshioka H, Inagawa $\mathrm{T}$, Tokuda $\mathrm{Y}$, Inokuchi F: Chronic hydrocephalus in elderly patients following subarachnoid hemorrhage. Surg Neurol 53: 119-125, 2000 